\title{
BMJ open 'It's on my iPhone': attitudes to the use of mobile computing devices in medical education, a mixed-methods study
}

\author{
Sean Wallace, Marcia Clark, Jonathan White
}

To cite: Wallace $\mathrm{S}$, Clark M, White J. 'It's on my iPhone': attitudes to the use of mobile computing devices in medical education, a mixed-methods study. BMJ Open 2012;2: e001099. doi:10.1136/ bmjopen-2012-001099

- Prepublication history and additional material for this paper are available online. To view these files please visit the journal online (http://bmjopen.bmj.com).

Received 21 March 2012 Accepted 6 July 2012

This final article is available for use under the terms of the Creative Commons Attribution Non-Commercial 2.0 Licence; see http://bmjopen.bmj.com

Department of Surgery, University of Alberta, Edmonton, Alberta, Canada

Correspondence to Dr Sean Wallace; sean.wallace@ualberta.ca

\section{ABSTRACT}

Objective: The last decade has seen the introduction of new technology which has transformed many aspects of our culture, commerce, communication and education. This study examined how medical teachers and learners are using mobile computing devices such as the iPhone in medical education and practice, and how they envision them being used in the future.

Design: Semistructured interviews were conducted with medical students, residents and faculty to examine participants' attitudes about the current and future use of mobile computing devices in medical education and practice. A thematic approach was used to summarise ideas and concepts expressed, and to develop an online survey. A mixed methods approach was used to integrate qualitative and quantitative findings.

Setting and participants: Medical students, residents and faculty at a large Canadian medical school in 2011.

Results: Interviews were conducted with 18 participants (10 students, 7 residents and 1 faculty member). Only 213 participants responded to the online survey (76 students, 65 residents and 41 faculty members). Over $85 \%$ of participants reported using a mobile-computing device. The main uses described for mobile devices related to information management, communication and time management. Advantages identified were portability, flexibility, access to multimedia and the ability to look up information quickly. Challenges identified included: superficial learning, not understanding how to find good learning resources, distraction, inappropriate use and concerns about access and privacy. Both medical students and physicians expressed the view that the use of these devices in medical education and practice will increase in the future.

Conclusions: This new technology offers the potential to enhance learning and patient care, but also has potential problems associated with its use. It is important for leadership in medical schools and healthcare organisations to set the agenda in this rapidly developing area to maximise the benefits of this powerful new technology while avoiding unintended consequences.

\section{INTRODUCTION}

The last decade has seen the introduction of new technology which has transformed many

\section{ARTICLE SUMMARY}

Article focus

- The rapid adoption of new technology has transformed many aspects of our culture, commerce and communication, and has the potential to change the way we teach, learn and practice medicine in the future.

- This study examined how medical teachers and learners are using mobile computing devices such as the iPhone ${ }^{\mathrm{TM}}$ in medical education and practice, and how they envision them being used in the future.

Key messages

- Mobile computing devices will soon be ubiquitous in clinical environments.

- This new technology offers the potential to enhance learning and patient care, but also has potential problems associated with its use.

- It is important for policy-makers to start a dialogue with users to understand their needs, and to maximize the benefits of this powerful new technology while avoiding unintended consequences.

Strengths and limitations of this study

- The study population was large, and response rates were relatively low; we cannot exclude an element of response bias.

- Despite this, we demonstrated that use of the devices is widespread among students and residents and is relatively common among faculty. We believe that examining the behaviours and attitudes of a small group of "early adopters" is useful in understanding how these devices may be employed by a larger number of users in the future.

aspects of our culture, commerce, communication and education. Mobile computing devices such as the iPhone and the iPad have been rapidly adopted in many countries, providing access to information in ways that were not possible before. ${ }^{1}$ We believe that mobile access to information made possible by these devices has the potential to change how medicine is learned and practised now and in the future. 
Most previous studies on the use of mobile computing devices in medical education have focused on the use of the personal digital assistant (PDA). ${ }^{2-4}$ These studies demonstrated that PDAs could be used by medical students in a variety of ways: to $\log$ their experiences, to access information about medical conditions and drug treatment, to perform calculations and make basic notes. Provision of these functions has been shown to enhance student learning in the clinical environment and to increase student knowledge scores. ${ }^{5} 6$

Recent work has focused on the current generation of mobile computing devices in which multiple functions are integrated into one device. For the purposes of this study, we define a 'mobile computing device' as a handheld device which provides constant connection to the internet via email, text messaging, video-conferencing and social networking software, often integrated with additional functions such as a camera; the devices can also be used to access multimedia content such as podcasts and video. ${ }^{7-11}$ This definition includes all manner of 'smartphones' such as the iPhone and the Blackberry and devices such as the iPad but excludes desktops, netbooks and laptop computers. Applications, or 'apps', are programmes developed to run on a device for a specific purpose ${ }^{12}$; there are hundreds of thousands of applications available in a wide range of categories including some tailored to specific medical fields, such as infectious disease and neurosurgery. ${ }^{12}{ }^{13}$ Released in April 2010, the Apple iPad is a tablet computer that operates similar to the iPhone with a faster processor and a larger screen. ${ }^{14}$ Immediately upon release, debate surrounded the use of smartphones as a clinical tool, with opinions ranging from overwhelming support to stark opposition. ${ }^{15}$ Many authors have been enthusiastic about the use of such devices in medical education and in practice, ${ }^{16} 712$ but few have examined the current use of these devices and considered the ways in which medical learners and teachers are adopting the devices into everyday practice.

The aim of this study was to establish how learners and teachers at our medical school are currently using mobile devices and to identify features of their use to assist with the development of policy that will direct the use of mobile computing devices in the years ahead.

\section{METHODS}

Ethics approval was obtained from the local Health Research Ethics Board. Semistructured interviews were conducted with medical students, residents and faculty at our school about participants' current use of mobile devices in medical education. Interviews were held in June 2011, were 30-45 min in length and were conducted by SW, a male research assistant on the project, who has completed a degree in Psychology. Interview questions were developed by all authors after a review of the literature (appendix 1). The interviews were conducted in person (with the exception of one phone interview) at a private office space located in our medical school.
Convenience sampling was used: participants were contacted through email, inviting voluntary participation in an interview. All medical students, residents and faculty at the school were included in the invitation email distribution list and a single reminder email was sent. There were no specific exclusion criteria. No repeat interviews were conducted. Consent was obtained in person by the interviewer. The interviews were designed to elicit information about participants' current use of mobile computing devices, and their opinions on the current and future use of the devices in medical education. Interviews were audio-recorded and transcribed by the primary author, and compiled with field notes taken during the interviews. For the majority of participants, no relationship was established prior to the study, and the researchers' personal goals or reasons for conducting were not revealed to participants. No feedback regarding the interviewer characteristics was elicited, and transcribed interviews were not returned to participants for feedback. No participants withdrew from the study. Transcripts and field notes were analysed by two of the authors (SW and JW) using a thematic approach to identify emergent ideas and concepts expressed by participants and ensure that data saturation had been achieved.

Themes identified from a review of the literature and interview data were used to construct an anonymous 17-question online survey that was then sent to all medical students ( $\mathrm{n}=650$ approximately), residents ( $\mathrm{n}=900$ approximately) and faculty (n=1000 approximately) at our institution (appendix 2). Items included Likert agree/disagree questions, dichotomous choice (yes/no), single-best response items and free-response items. The study was piloted with a group of student volunteers $(n=6)$ before distribution. Convenience sampling was used: participants were contacted through email, inviting voluntary participation in the survey. All medical students, residents and faculty at the school were included in the email distribution list for the online survey and a single reminder email was sent. There were no specific exclusion criteria. Participants who had been interviewed were free to respond to the survey too. Mixed methods analytical techniques were then used to synthesise qualitative results from the interviews (themes and quotations) with quantitative results from the online survey (number of respondents in agreement with particular statements).

\section{RESULTS}

Interviews were conducted with 18 participants (10 medical students, 7 residents and 1 faculty member); 17 of these reported current use of a mobile computing device. Only 213 participants responded to the online survey (93 medical students, 72 residents and 48 faculty members). All of the major specialities were represented among residents and faculty: family medicine, paediatrics, psychiatry, surgery and its specialities, internal medicine and its specialitites, anaesthesiology, infectious disease, obstetrics and 
gynaecology, intensive care, occupational medicine, emergency medicine, physical medicine and rehabilitation. In the following section, interview quotations are presented to highlight particular themes identified, while percentages in brackets are used to indicate the proportion of respondents to the online survey who expressed agreement with a particular theme or statement observed at interview. Participants are identified as student (S), resident $(\mathrm{R})$ or faculty member $(\mathrm{F})$.

\section{Patterns of usage}

Data from the interviews suggested that the use of mobile computing devices was widespread and frequent, and that it occurred in all settings where learners and teachers were present, from classrooms to hospitals. Almost $90 \%$ of participants reported current use of a mobile computing device (overall: $87 \%$, students $85 \%$, residents $90 \%$ and faculty $85 \%$ ), with the Apple iPhone being the most prevalent (75-80\% in each group). The number of devices used were as follows: iPhone 126, Blackberry 19, Android 12, HTC 1, Samsung 1, Palm 2, Symbian 1 and multiple devices 1 . A total of $85 \%$ of participants owning a mobile computing device reported using it at least once a day for medical purposes (students $85 \%$, residents $98 \%$ and faculty $65 \%$ ).

In my entire class everybody has a smartphone ... I'm probably one of the last ones to be getting an iPhone. (R02)

\section{Advantages}

Participants listed a number of advantages associated with the use of mobile computing devices: portability, fast access to information on the internet, efficient use of time, flexible communications, powerful applications and access to multimedia resources.

I bring my laptop to work now to study and it's way too heavy and cumbersome ... with the iPad I can just carry the size of a small textbook and have access to multiple textbooks. (R05)

Participants focused on having rapid access to resources required for learning or clinical care 'on the go' and stated that, in general, having access to a mobile computing device had a positive educational effect for them (students 55\%, residents $95 \%$ and faculty $75 \%$ ).

Participants provided further information on how they used mobile computing devices to 'look stuff up'. A total of $41 \%$ of respondents described using their devices to look up unfamiliar terms or conditions at least once in the normal course of each day (students: $48 \%$ more than once per day, residents $55 \%$ more than once per day and faculty: $25 \%$ at least several times per week).

I would use it every time I had a new admission ... and then on the wards maybe 2-3 times a day, particularly if I didn't know a certain drug or I wasn't sure of the dose. (R07)
I intend to use it a lot next year in my clerkship year when

I don't know something I can look it up quickly. (S10)

Respondents described the immediacy and convenience of accessing information right at the time when it was needed:

I think that it's always best learning when you look things up right away ... you don't have to wait, because if you wait until the end of the day and look everything up things get dropped off the list. (R01)

Others described the use of 'looking stuff up' as a way of double-checking their own knowledge:

I'm quite rusty so I'm always double checking ... and where I was working I had the leisure of time to look things up and not someone to ask. (R06)

Participants described the use of the devices in three major domains: information management, communication and time management.

\section{Information Management}

Respondents described accessing a number of information sources using the devices. These included online textbooks (students $70 \%$, residents $67 \%$ and faculty $35 \%$ ), medical podcasts (students $60 \%$, residents $38 \%$ and faculty 23\%), medical calculators (students $75 \%$, residents $98 \%$ and faculty $75 \%$ ) and online lectures (students $50 \%$, residents $17 \%$ and faculty $17 \%$ ). Note taking was a commonly described use (students $45 \%$, residents $67 \%$ and faculty $50 \%$ ). Both the interview and survey found that defining unfamiliar terms was a common practice (students $93 \%$, residents $95 \%$ and faculty $82 \%$ ), with Google (50\%) and Wikipedia (15\%) being among the most popular sources. A number of other uses were also described, including the use of the devices for accessing medical journal websites $(60 \%)$ and getting medical news online $(74 \%)$.

'I like looking up stuff on Wikipedia-it's quick and easy to access. It's not something I would base patient care on but if it's something like 'what class of drug is this?' then it's useful to find that answer.' (S03)

Seventy seven per cent of participants indicated that they used at least one medical 'app' regularly. Participants described a broad range of 'apps' including medical references, calculators and programme-specific 'apps'; Epocrates, Pepid, Medscape, MedCalc, Lexicomp, Skyscape and Dynamed were the most popular applications. Over $50 \%$ of participants reported using their favourite 'app' at least once a day.

\section{Communication}

More than $80 \%$ of respondents described the use of the devices to communicate with peers, teachers and other members of the healthcare team about patient care using email, telephone and text messages. A total of 
$70 \%$ of residents and $45 \%$ of faculty described texting students about patient care.

(Phone buzzes) See this is exactly it here, I just got a text about a patient. (R05)

Many participants described texting as a more efficient way of communicating than meeting in person or talking on the telephone:

(regarding texting) I prefer that to paging because you can just wait until you get a response, you can ask specific questions, you don't have to sit by a phone and wait 10 minutes until they have time. (R02)

Although many participants described using their devices to communicate about patient care, less than $10 \%$ reported using the devices to transmit or view clinical data or images relating to patients (students $10 \%$, residents $9 \%$ and faculty 5\%). Respondents also described using their devices to respond rapidly to email, and to keep up with the flow of communication so that they did not 'get behind'.

The people in our class who don't have smartphones have missed out on a lot of things because they're time dependent. (S02)

\section{Time management}

Participants commonly used the devices to manage their time, by accessing schedules and calendars 'on the go' (students $81 \%$, residents $84 \%$ and faculty $56 \%$ ).

I use it to get my class schedule ... since our scheduling locations change everyday. (S09)

I communicate with other residents, we all share calendars that we all can get on our smartphones... (including) our teaching calendar and extracurricular calendar... we can share events, academic half-days, or maybe other days of interest to each other. (R06)

A number of participants described using their devices to keep a record of their clinical experiences $(27 \%)$. Some participants described using the devices to make use of 'downtime' to learn more efficiently:

All the residents in my program pass around flashcards that they made for themselves for studying and it's great for like on the bus, or waiting around, just quick little memorization tools. (R01)

\section{Challenges}

Participants identified a number of potential challenges to the use of mobile computing devices in medical education.

\section{Superficial learning}

Some participants described their worry that these devices might enable learners to access information so rapidly that it may inhibit the internalisation of knowledge which is a traditional part of medical education, leading to a potential for 'superficial learning'.

I think in a way perhaps you look something up and you only get bites of things ... I think it might be promoting a more superficial knowledge of things as opposed to an in-depth knowledge of things. (F01)

You can end up relying on it ... rather than memorizing all the terms and having a good differential you can always just pull up a list. (S07)

I use my phone all time, it's ridiculous ... I never know everything (R05)

\section{Trusting information sources}

Learners expressed concerns about how they should navigate the information available on the devices, how they should find 'good applications', and how they would know if the information provided was of high quality. Only $47 \%$ of participants agreed with the statement: 'I feel as though I don't know enough about what is out there to effectively use my smartphone' (students $54 \%$, residents $31 \%$ and faculty $57 \%$ ). A majority of participants also expressed that they could be using the technology more effectively than they are currently (students $67 \%$, residents $76 \%$ and faculty $60 \%$ ).

Not knowing really what is out there... having a list of what's recommended, that would probably be the most helpful. (S03)

One of the biggest limitations is just trying to find (sources) that are good and reliable, because I don't like using Wikipedia; it's good for definitions and stuff but I don't like relying on it for treatments $(\mathrm{S} 07)$

\section{Distraction}

Participants provided accounts of mobile computing devices causing distraction from normal activities of learners and physicians in classrooms and clinical settings. Sixty-three per cent of respondents agreed with the statement: 'Smartphones are a distraction in the classroom' (students $66 \%$, residents $50 \%$ and faculty $74 \%$ ).

The use within the classroom really can be a bit distracting because it's too easy to flip onto Facebook ${ }^{\mathrm{TM}}$. (S04)

It's very positive outside the classroom for studying. Inside the classroom it's starting to go maybe a little overkill. (S04)

Some also considered the technology a distraction in the clinical environment (students $33 \%$, residents $24 \%$ and faculty $46 \%$ ):

You're not really paying as close attention as you could be if you're always looking stuff ... like we were on rounds this morning ... and I was too busy looking up how to spell 
one of the drugs and what it did ... I just managed to jot down but didn't get to hear anything else about it. (S07)

There's always going to be those people that are messing around on Facebook ${ }^{\mathrm{TM}}$ on their iPhone when they should be attending rounds. (R02)

\section{Concerns about information privacy}

Participants expressed concern about potential implications for privacy and confidentiality when material relating to patients is held on one's personal device. About half of participants described using their device to take a picture of a patient (students $33 \%$, residents $58 \%$ and faculty $67 \%$ ):

I think there's problems having personal stuff and professional stuff on the same device (S03)

If you're accessing patient information I think that's one thing that has to be addressed for sure. (R04)

Approximately $34 \%$ of participants agreed that concerns over privacy were affecting their own use of the devices (students $25 \%$, residents $31 \%$ and faculty $51 \%$ ).

The only problem is with all these smartphones is privacy, how much are we going to be communicating patient information? (R05)

\section{Blurring of personal/professional boundaries}

Participants also expressed concerns about mobile computing devices allowing potential intrusion of personal matters (eg, personal text messages) into professional and clinical duties. Some anticipated having separate devices for personal and professional uses.

One way to do it though is ... having my own personal phone versus having (a device specifically for patient care) ... I know it'll be secure and safe, I don't have to worry about the technical upkeep of everything. (R04)

Concern that use of this technology had the potential to compromise professional behaviour was also expressed (students $37 \%$, residents $27 \%$ and faculty $10 \%$ ).

Sometimes it can be taken as 'are you texting someone else, are you doing something personal'? Others on the team may not see you taking notes that way, so sometimes it can be a disruptive thing. (R04)

Participants also expressed concern about learners' ability to access mobile computing devices; $40 \%$ identified cost as a limiting factor in adoption of the technology (students $43 \%$, residents $40 \%$ and faculty $40 \%$ ):

I would've upgraded to an iPhone earlier but I didn't have the money ... you have to remember that people that can't afford this are actually becoming disadvantaged. (R02)

Participants suggested that some policy may be required to streamline use across different systems and to provide guidance on the proper professional use of these devices. Forty-seven per cent agreed with the statement: 'university policy regarding smartphone usage in medical education would be beneficial' (students 60\%, residents $29 \%$ and faculty $51 \%$ ).

\section{Anticipated future uses}

The majority of participants (96\%) agreed with the statement: 'smartphones will increase in usage in the future of medical education' (students 95\%, residents 99\% and faculty $89 \%$ ). Many participants anticipated that these devices will soon replace the use of traditional textbooks, and that they would soon be used as a routine part of clinical care:

I see it replacing textbooks completely. (R04)

I think we'll all be using them, I don't think anybody will be able to go without because they're so useful. (R03)

Some participants described desired functionalities that have yet to be developed on the devices, such as prescribing and receiving 'automatic updates' about patients. Participants also envisaged an increased integration of devices into patient care, and anticipated a replacement of traditional medical records:

Security issues are going to be huge but if they can get that sorted out I think that'll help it replace the medical chart to a certain degree. (R06)

I wouldn't be surprised if say 5 years from now everyone has their iPad or something like that with access to chart information right on there so you can stay right up with it... $(\mathrm{S} 07)$

\section{DISCUSSION}

This study confirms that mobile computing devices such as the iPhone are widely used by medical learners and teachers at our school for everyday communication and information management relating to patient care and education. Use of the devices was described in a wide range of clinical environments, and it seems likely that their presence will soon be ubiquitous. ${ }^{17}{ }^{18}$ Participants in this study described integrating this technology into patient care, learning and work. The technology provides rapid access to information and facilitates communication between team members, and has the potential to enhance both patient care and learning. Some authors have written enthusiastically about how these devices will enhance learning, ${ }^{7}$ and other have suggested they have much potential for patient education ${ }^{14}$ and mobile access to patient records. ${ }^{19}$ Others have called for the development of learning resources specifically designed to take advantage of the devices' features. ${ }^{20}$ There are already a large number of 'apps' available for those engaged in patient care and medical education, and more are being developed all the time. ${ }^{21}$

Rapid uptake of a new technology may have unanticipated consequences, including disruption of the normal 
practices of medical education and patient care. Unexpected effects observed in this study included the potential for distraction and superficial learning, and concerns over professionalism and privacy. These devices allow information to permeate everywhere-the medical student carrying an iPhone can be the recipient of direct phone and video calls, text messages, instant messages and emails at any time the device is active. Technologies that allow us to be 'always-on' may prevent us from giving full attention to tasks at hand, and may distract users from the normal activities of teaching, learning and working. Some have even suggested that new technologies are changing the way that our brains process information, making us 'jet-skiers' skimming across the surface of the sea of information instead of 'scuba-divers' who take time to descend more deeply. ${ }^{22}$ Our relationship with information is changing, requiring us to develop more discipline about how we manage it. ${ }^{23}$ Others have raised concerns about the potential for distraction during patient care that mobile devices provide, ${ }^{24-26}$ and interruptions during tasks have been associated with an increased rate of error and partial task completion. ${ }^{27} 28$ The findings of this study support those of Wu $e t a l^{29}$ who employed an ethnographic approach to examine the communication patterns of physicians and residents using smartphones. While communication appeared to be made more efficient by smartphones, the authors observed that some physicians using the devices appeared to be 'more globally connected but less locally present'. The technology also facilitated the interruption of normal activities, and was observed to detrimentally affect team relationships; coworkers preferred communication in person to texting, and objected to interruption of important conversations and patient care by calls and messages on the device.

This study supports the findings of Davies $e t a l,{ }^{22}$ who recently conducted a study examining the use of PDAs by medical students. In common with our findings, this study showed that smartphones facilitated student learning by providing timely access to key facts, allowing learning in context and by repetition, supplementing other ways of learning and making use of wasted time. The study also suggested that barriers to the use of these devices included interruption to clinical experiences, and lack of buy-in from teachers and patients.

Our findings also raise questions about the way these devices may change the 'location of knowing.' Medical education has traditionally been focused on the internalisation, management and apply a large amount of knowledge 'in the head' of the practitioner. Trainees are deemed competent for independent practice when they can handle a problem 'on their own'; that is, having internalised the knowledge relevant to a particular set of problems, they can independently access it, apply it to a patient and assess its effects. The advent of mobile devices such as the iPhone leads to another possibility: that the practitioner may not have to internalise much knowledge at all, if they have a device that can provide access to the relevant information at the right time from a reliable source. ${ }^{17}$ This would constitute a major shift in professional identity: 'I don't know what to do, but I know where to look to find out'. This concept has been described as 'asking The Cloud. ${ }^{30}$ It seems unlikely that using 'The Cloud' will ever completely replace the need to internalise medical knowledge, but as technology advances and the scope of medical knowledge continues to expand, we may become more dependent on mobile computing devices to find up-to-date, evidence-based information relevant to patient care. Being able to 'know where to look when I don't know' is likely to become a vitally important skill for all medical practitioners. ${ }^{16}$ Medical students would be expected to benefit from training to ensure that they can efficiently navigate 'The Cloud' to find the information they will need to function. ${ }^{4}$

Unfettered access to information may also blur the boundaries between personal and professional information. The use of these devices may lead to interesting professional dilemmas: if you are with a patient, should your device be turned off? If your partner texts you during a consultation with a patient, is it OK to read the message? What should you do if a friend accidentally sees a "clinical photo' or a text relating to a patient on your phone? Some of our participants advocated the use of separate devices for 'work' and 'personal life', and others have suggested that healthcare organisations may eventually provide mobile devices intended for use at work only. ${ }^{9} 15$ The speed of adoption of this technology has been impressive; users of mobile computing devices appear to be running ahead of leaders, policy-makers and educators. The technology that drives the devices is not going away, and will probably continue to advance and accelerate. ${ }^{20}$ Users will continue to find new and unexpected uses for the devices, and healthcare organisations will continue to integrate these devices into patient care. ${ }^{17}$ Others have written about the need for 'early adopters' of the devices among physicians to act as role models, demonstrating appropriate use to their students. ${ }^{31}$

Our study was limited in several areas. First, response rates to the online survey were relatively low, about $15 \%$ for students, $10 \%$ for residents and $7 \%$ for faculty. Second, response bias is likely: non-users of the technology would be anticipated to be less likely to respond to a survey about mobile computing devices. Despite these limitations, recurring themes were clearly established, and our demographic included a wide diversity of residency programmes, students and faculty. Even though our findings cannot be widely generalised, we believe that use of the devices is widespread among students and residents and is relatively common among faculty. We also believe that examining the behaviours and attitudes of a small group of 'early adopters' is useful in understanding how these devices may be employed by a larger number of users in the future. 
This study has encouraged a debate among students and faculty about the use of mobile computing devices at our medical school, and we intend to develop a set of guidelines to help learners and teachers responsibly use these devices as the technology develops further. We intend to conduct further studies on other aspects of the use of these devices which were only touched upon in this initial study: for example, the effects of early or late adoption of the devices by learners and the effects of the devices on patterns of communication and information-sharing among healthcare providers.

All those engaged in health professions education need to make themselves aware of the potential of these devices to enhance learning and patient care, and of the potential problems associated with their use. We encourage educators to embrace this new technology, to study its further adoption and to assist with the responsible integration of these devices into medical education.

\section{CONCLUSIONS}

Mobile computing devices have been rapidly adopted by medical learners and teachers at our school, and it seems likely that their presence will soon be ubiquitous. This new technology offers the potential to enhance learning and patient care, but also has potential problems associated with its use, and may redefine how we manage information in medicine. It is important for leadership in medical schools and healthcare organisations to set the agenda in this rapidly-developing area, to start a dialogue with users to understand their needs and to maximise the benefits of this powerful new technology while avoiding unintended consequences.

Acknowledgements Funding for this project was provided by a University of Alberta Faculty of Medicine and Dentistry summer studentship 2011. We would like to acknowledge the assistance of Ms Shannon Erichsen with this project.

Contributors All three authors are justifiably credited with authorship, according to the authorship criteria. In detail: SW: collection, analysis and interpretation of data, interviewer, drafting the article, final approval of the version to be published. MC: study conception and design, revising the article, final approval of the version to be published. JW: study conception and design, analysis and interpretation of data, revising the article, final approval of the version to be published.

Competing interest All authors have completed the Unified Competing Interest form at www.icmje.org/coi_disclosure.pdf (available on request from the corresponding author) and declare that (1) (initials of relevant authors) have support from (name of company) for the submitted work; (2) (initials of relevant authors) have (no or specified) relationships with (name of companies) that might have an interest in the submitted work in the previous 3 years; (3) their spouses, partners, or children have (specified) financial relationships that may be relevant to the submitted work; and (4) (initials of relevant authors) have no (or specified) non-financial interests that may be relevant to the submitted work.

Ethical approval Ethics approval was obtained from the University of Alberta Health Research Ethics Board (ref: 00023167).

Data sharing statement Technical appendix, statistical code and dataset available from the corresponding author at seanwallace1@gmail.com or jswhite1@ualberta.ca.

\section{REFERENCES}

1. Franko OI, Tirrell TF. Smartphone app use among medical providers in ACGME training programs. J Med Syst Published Online First: 4 November 2011. doi:10.1007/s10916-011-9798-7

2. Ho K, Lauscher HN, Broudo M, et al. The impact of a personal digital assistant (PDA) case log in a medical student clerkship. Teach Learn Med 2009;21:318-26.

3. Kurth RJ, Silenzio V, Irigoyen MM. Use of personal digital assistants to enhance educational evaluation in a primary care clerkship. Med Teach 2002;24:488-90.

4. Lasserre K, Eley D, Baker P, et al. Medical students out of town but not out of touch: use of personal digital assistants to improve access to clinical information and enhance learning at the point of care in rural and remote Australia. Aust J Rural Health 2010;18:87-8.

5. Farrell MJ, Rose L. Use of mobile handheld computers in clinical nursing education. J Nurs Ed 2008;47:13-19.

6. Sutton J, Stockton L, McCord G, et al. Handheld computer use in a family medicine clerkship. Acad Med 2004;79:1114-19.

7. Dala-Ali BM, Lloyd MA, Al-Abed Y. The uses of the iPhone for surgeons. The Surgeon 2011;9:44-8.

8. Luanrattana R, Win KT, Fulcher J, et al. Mobile technology use in medical education. J Med Syst. 2012;36:113-22.

9. Phillippi JC, Buxton M. Web 2.0: easy tools for busy clinicians. J Midwif Women's Health 2010;55:472-6.

10. Zolfo M, Iglesias D, Kiyan C, et al. Mobile learning for HIV/AIDS healthcare worker training in resource-limited settings. AIDS Res Ther 2010;7:35.

11. White JS, Sharma N, Boora P. Surgery 101: evaluating the use of podcasting in a general surgery clerkship. Med Teach 2011;33:941-3.

12. Kubben P. Neurosurgical apps for iPhone, iPod Touch, iPad and Android. Surg Neurol Int 2010;1:89.

13. Oehler RL, Smith K, Toney JF. Infectious diseases resources for the iPhone. Clin Infect Dis 2010;50:1268-74.

14. Berger E. The iPad: gadget or medical godsend? Annals Emerg Med 2010;56:A21-2.

15. Hagland M. Handheld juggernaut. Healthcare Informatics 2010;27:8, 12-14.

16. Busis N. Mobile phones to improve the practice of neurology. Neurologic Clinics of NA 2010;28:395-410.

17. Brusco JM. Using smartphone applications in perioperative practice. AORN J 2010;92:503-8.

18. Baumgart DC. Smartphones in clinical practice, medical education, and research. Arch Intern Med 2011;171:1294-6.

19. Gamble KH. Wireless Tech Trends 2010. Trend: smartphones. Healthc Inform 2010;27:24, 26-7.

20. Trelease RB. Diffusion of innovations: smartphones and wireless anatomy learning resources. Anat Sci Ed 2008;1:233-9.

21. Visser BJ, Bouman J. There's a medical app for that. BMJ Careers Published Online First: 18 April 2012. http://careers.bmj.com/ careers/advice/view-article.html?id=20007104

22. Carr N. The shallows: what the internet is doing to our brains. W. W. Norton \& Company, New York City, NY, 2011.

23. Johnson CA. The information diet: a case for conscious consumption. O'Reilly Media, Sebastapol, CA, 2012.

24. Davies BS, Rafique J, Vincent TR, et al. Mobile Medical Education (MoMEd)-how mobile information resources contribute to learning for undergraduate clinical students-a mixed methods study. BMC Med Educ 2012;12:1.

25. Richtel M. As doctors use more devices, potential for distraction grows. NY Times, New York City, NY, 2011.

26. Smith T, Darling E, Searles B. 2010 Survey on cell phone use while performing cardiopulmonary bypass. Perfusion 2011;26:375-80.

27. Westbrook Jl, Woods A, Rob Ml, et al. Association of interruptions with an increased risk and severity of medication administration errors. Arch Intern Med 2010;170:683-90.

28. Westbrook JI, Coiera E, Dunsmuir WTM, et al. The impact of interruptions on clinical task completion. Qual Saf Health Care 2010;19:284-9.

29. Wu R, Rossos P, Quan S, et al. An evaluation of the use of smartphones to communicate between clinicians: a mixed-methods study. J Med Internet Res 2011;13:e59.

30. Mallikharjuna Rao NM, Sasidhar C, Satyendra Kumar V. Cloud computing through mobile-learning. Int J Adv Comp Sci Applic 2010;1:42-7.

31. Putzer GJ, Park Y. The effects of innovation factors on smartphone adoption among nurses in community hospitals. Perspect Health Inform Manag 2010;1:1b. 